\title{
Erratum
}

\section{Electrophilic Functionalization of Non-Activated Olefins Catalyzed by Lewis Superacids}

Sylvain Antoniotti, Sophie Poulain-Martini, Elisabet Duñach* Synlett 2010, 2973.

In the advance online publication (e-First) version of this article the graphical abstract did contain a mistake. This has been corrected for both the current online version and the print publication. We apologize for this error. 